\title{
The Visual Web User Interface Design in Augmented Reality Technology
}

\author{
Chouyin Hsu \\ Department of Information Management \\ Overseas Chinese University \\ Taichung, Taiwan
}

\author{
Haui-Chih Shiau \\ Department of Information Technology \\ Overseas Chinese University \\ Taichung, Taiwan
}

\begin{abstract}
Upon the popularity of 3C devices, the visual creatures are all around us, such the online game, touch pad, video and animation. Therefore, the text-based web page will no longer satisfy users. With the popularity of webcam, digital camera, stereoscopic glasses, or head-mounted display, the user interface becomes more visual and multi-dimensional. For the consideration of 3D and visual display in the research of web user interface design, Augmented Reality technology providing the convenient tools and impressive effects becomes the hot topic. Augmented Reality effect enables users to represent parts of the digital objects on top of the physical surroundings. The easy operation with webcam greatly improving the visual representation of web pages becomes the interest of our research. Therefore, we apply Augmented Reality technology for developing a city tour web site to collect the opinions of users. Therefore, the website stickiness is an important measurement. The major tasks of the work include the exploration of Augmented Reality technology and the evaluation of the outputs of Augmented Reality. The feedback opinions of users are valuable references for improving $A R$ application in the work. As a result, the $A R$ increasing the visual and interactive effects of web page encourages users to stay longer and more than $80 \%$ of users are willing to return for visiting the website soon. Moreover, several valuable conclusions about Augmented Reality technology in web user interface design are also provided for further practical references.
\end{abstract}

Keywords- Visual Represeantion; User Interface Design; Augmented Reality; Google SketchUp

\section{INTRODUCTION AND RESEARCH BACKGOUND}

With the popularity of webcam, digital camera, stereoscopic glasses, or head-mounted display, the user interface becomes more visual and diverse. It's an interesting time to be working on the visual web user interface now. The web pages evolved from a text-based system to the current rich and interactive medium that supports images, 2D graphics, audio and video. There is no question of the coolness of Augmented Reality Technology. We have seen many worldclass brands like Lego, Star Wars, and Avengers commercialize the technology. Many Enterprises start to pursue developing the web sites based on Augmented Reality technology.

It was in the late 60's that researchers started to describe how and where people interacted with technology. The term 'augmented reality,' however, was not coined until the early 90's, when airplane manufacturer Boeing started using augmented reality goggles to assist engineers in the airplane assembling process. Recently, Augmented Reality enables you to represent parts of the digital world on top of the physical world. Augmented Reality is a hot topic that is growingly attracting attention of the developers of web user interface. This is sometimes reinforced by the fact that available tools for 3D UIs are toolkits, interface builders, rendering engines, etc. Moreover, rendering skill is mostly used in the work for increasing the visual representation. As a result, the user interaction and visual design in 3D are becoming more and more popular.

The research work increases the visual web page design in Augmented Reality. The purpose is to explore the feasibility of Augmented Reality in web page design. The web page design skill with text-based, image-based, flash-based design is no longer popular. With the extremely increasing new users of online games, the visual effect becomes an important element of web page design since the users are used to the visual representation on the screen. The research work builds a city tour web site in Augmented Reality tasks. We also do the evaluation for collecting the opinions of users for modifying the research direction. Therefore, the research result contains the skillful comments of Augmented Reality tools as well as the practical suggestion for further visual web page design.

\section{RELATED WORK}

The webcam and mobile phone and diverse devices have led to a complete redesign on the traditional User Interface (UI). There is a new emphasis on intuitive ease of use. Moreover, the development of 3D User Interfaces (UIs) mostly remains an art more than a principled-based approach.

Several methods have been introduced to decompose this life cycle into steps and sub-steps, but these methods rarely provide the design knowledge that should be typically used for achieving each step [1][9][17].

\section{A. Visual User Interface Design}

User interface design or user interface engineering is the design of computers, appliances, machines, mobile communication devices, software applications, and websites with the focus on the user's experience and interaction. Web user interface focuses on the web page design to link users and web content.

Visual user interfaces have been regarded as one of the most promising routes. They exploit powerful human vision 
and spatial cognition. Carefully designed visual user interfaces can shift the user's mental load from slow reading to faster perceptual processes such as visual pattern recognition. Visual interfaces should support information exploration and bring users' attention [5][8].

Text-based web page is not suitable for the current modern users. Therefore, visual presentation is an important topic. More visual and interactive web operations are more crucial for web pages. With the stimulation of 3D tools, Augmented Reality tools are used for improving the presentation of web content. Unlike images or video, the user can operate and control the display of web objects. Therefore, more relative research topics become popular.

\section{B. Augmetned Reality}

The goal of Augmented Reality is to add information and meaning to a real object or place. The field of Augmented Reality (AR) has existed for just over one decade, but the growth and progress in the past few years has been remarkable. In 1997, the first author published a survey [1][2] and defined the field, described many problems, and summarized the developments up to that point. Since then, the field has grown rapidly.

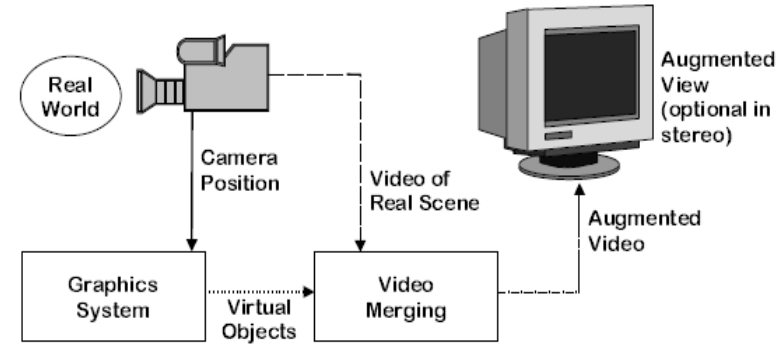

Fig. 1. The Explanation Of Augmented Reality Effect

Unlike virtual reality, augmented reality does not create a simulation of reality. Instead, it takes a real object or space as the foundation and incorporates technologies that add contextual data to deepen a person's understanding of the subject, as shown in Figure 1[26]. Many popular applications of AR successfully developed in various fields are explained as follows.

- Advertising and marketing: Marketers started to use AR to promote products via interactive AR applications. From Toyota to Nivea to Disney, a diverse array of brands has applied AR bandwagon for creating hype interaction with consumer.

- Task support: Complex tasks such as assembly, maintenance, and surgery can be simplified by inserting additional and useful information with AR technology. Some complicated repair works depend on AR information as well. Moreover, some restaurants provide the AR-enabled menu for users to 'see' the food first before they enjoy the food.

- Product design: AR can simulate planned products, including architecture, machine, car and more objects for better design discussion in advance.
- Navigation: AR can create virtual objects in museums and exhibitions, theme park attractions and books. The overlay between real and virtual worlds always makes novel navigation for users.

\section{Redering Technique}

Rendering is the final process of creating the actual 2D image or animation from the prepared scene. This can be compared to taking a photo or filming the scene after the setup is finished in real life. Several different, and often specialized, rendering methods have been developed. Rendering (computer graphics), generating an image from a model by means of computer programs. Many types of rendering are given as follows:

- 3D rendering, generating image or motion picture from virtual 3D models.

- High dynamic range rendering allows preservation of details that may be lost due to limiting contrast ratios.

- Non-photorealistic rendering, focuses on enabling a wide variety of expressive styles for digital art.

- Scanline rendering, algorithm for visible surface determination.

- Volume rendering, used to display a 2D projection of a 3D discretely sampled data set

Rendering engine has different types. Two major types are explained as follows.

- Game engine, system designed for the creation and development of video games.

- Web browser engine, software that takes information and displays the formatted content on the screen

\section{Evaluation Method}

The evaluation for user interface causes many discussions. From the perspectives of usability, the experts "inspect" your interfaces during formative evaluation and widely used in practice. Three major evaluation strategies which are considered to be used in the research are explained as follows:

- Heuristic Evaluation - Heuristic evaluations were introduced by Nielsen and Molich in 1990 in the influential paper "Heuristic Evaluation of User Interfaces" and later assembled as part of a book [23][27]. Usability inspection method for computer software that helps to identify usability problems in the user interface (UI) design. It specifically involves evaluators examining the interface and judging its compliance with recognized usability principles (the "heuristics"). An additional practical problem is that multiple usability experts should be used. It can often be more expensive and difficult to find many usability professionals.

- Cognitive Walkthroughs - A cognitive walkthrough is also a usability inspection method like heuristic evaluation but the emphasis is on tasks. The idea is basically to identify users' goals, how they attempt them in the interface, then meticulously identify problems 
users would have as they learn to use an interface. The method was also introduced at the same conference as Heuristic Evaluation (Lewis et al 1990). The cognitive walkthrough was an extension of earlier work by Polson and Lewis [15].

- Pluralistic Walkthroughs - A pluralistic walkthrough is a usability inspection method whereby representative users (normally played by the evaluators), developers and usability experts, step through a scenario, discussing the usability issues associated with each scenario step. Also known as storyboarding, the method involves the development of a series of tasks, presented to a panel of users in the form of hard-copy panels; the panelists are then asked to write down the actions they would take to complete the task [4].

\section{RESEARCH FLOW AND IMPLEMENTATION}

\section{A. Development Flow}

The research development flow containing four phases is explained as follows.

- The Concept phase - In the beginning, the concept of visual representation is the first idea. Then, the subject of web has to be decided. Not all the pages are suitable for visual effects. Therefore, the topic of city tour becomes the interesting work.

- The Implementation and Content Development phase Many web design tools and Augmented Reality tool are applied in the phase. Building models, rendering pictures, web page design and Augmented Reality effects are the major efforts in the phase. Google SketcUp and 3D Max are the tools for building models. It is pretty time consuming, but the output is really inspiring and exciting. The well-constructed models are helpful for generating good Augmented Reality effects.

- The User Interaction Phase - With the identification card of Augmented Reality, the user can control the representation of Augmented Reality on the screen. The output of real world and virtual objects combing together is really interesting for users.

- The Evaluation Phase - Heuristic evaluation is the most popular of the usability inspection methods. Heuristics Evaluations is done prior to and in addition to usertesting, not instead of user-testing. The feedback of evaluation is important for design improvement.

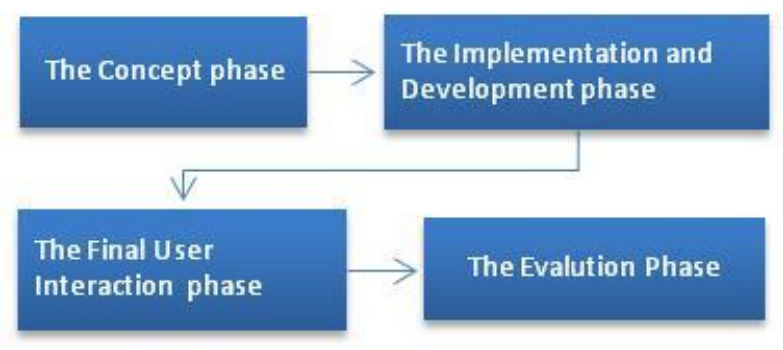

Fig. 2. The research development flow

\section{B. The Augmented Reality Implement and Research Ouput}

The index page of the web system is shown in Figure 3. It is the first image of the web to users. According to the opinions of users, the 3D-like, colorful and visual image brings lots of attention and they are attracted to stay on the site. The rendering technique is well applied for the effect. Rendering is the process of generating an image from a model, by means of computer programs. Many rendering software with SketchUp for rendering purposes are provided for different purposes, such light, motion or realism. There are Kerkythea, TrueSpace, Blender, Vue, and Podium are among the most popular rendering applications for SketchUp [28]. The rendering images used for the index page is one of successful designs in the work.

\section{TAICHUNG CITY TOUR 台中市導覽}

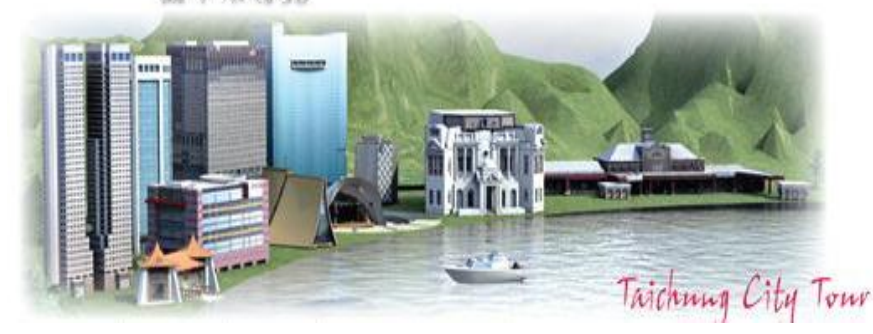

Fig. 3. The index page of the city tour web

Building models and make Augmented Reality effect are two major tasks. Googld Sketch Up and FLARTookit are used in the phases. Google SketchUp is a free, fun, easy-to-use 3D modeling application. Originally created for architects and designers, SketchUp is also a great tool for teaching geometry. FLARToolKit recognize the marker from input image, and calculate its orientation and position in 3D world. FLAToolKit has support for all major flash 3D graphics engine.

In order to explain the different effects of traditional 2Dlike picture and 3D-like Augmented Reality technology, an example of the Mid-Lake Pavilion of Taichung Park is given below.

The picture of the Mid-Lake Pavilion shown in Figure 4 displays the 'real' outlook of the building. In the other hand, the model of the Pavilion built with Google SketcUp given is given in Figure 5. According to the users' opinions, they spend more time to look the 3D-like model rather than picture. The model catches users' eyes immediately after they browse the web. The visual effect works again. We collect many opinions from users for improving the built models and page design.

Furthermore, the user will like to pay more attention to operate the Augmented Reality function. Since the webcam is easy to setup with the PC, the user can easily play with the Augmented Reality card to view the Pavilion model, as shown in Figure 6. The user can control the direction of the identification card to view the model from different angles and directions. With the Augmented Reality operation, the attached introduction information will possibly get the attention of users, as shown in Figure 7. As long as the user read the introduction, the major function of city tour website is herein fulfilled. 


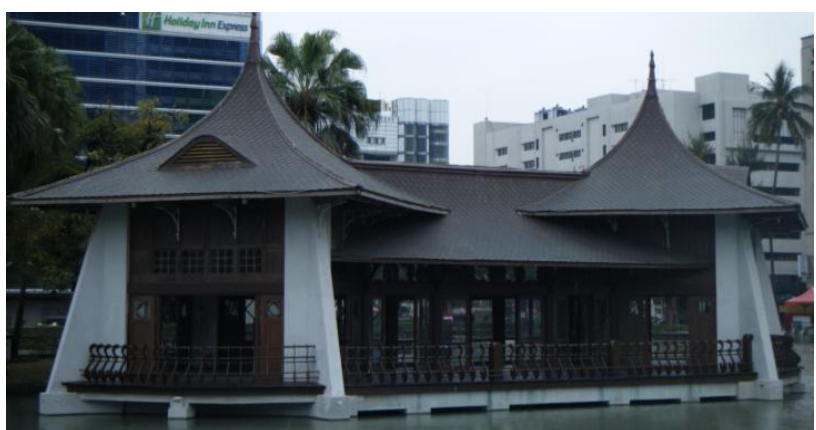

Fig. 4. The picture of the Mid-Lake Pavilion of Taichung Park

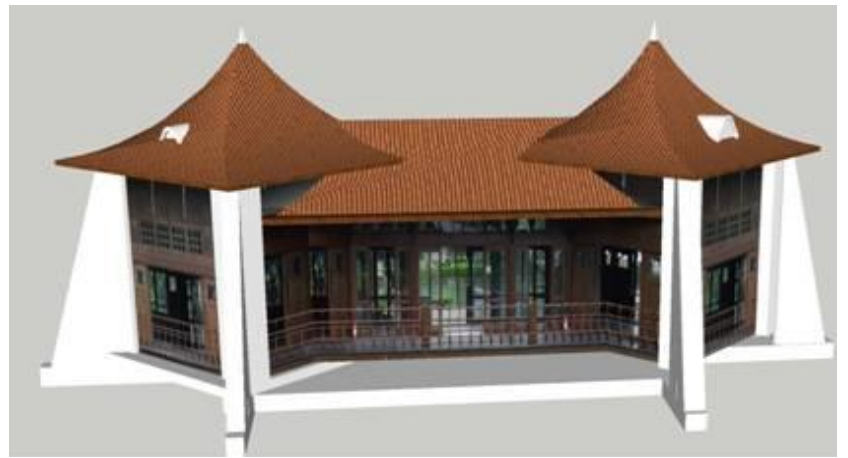

Fig. 5. The built model of the Mid-Lake Pavilion of Taichung Park

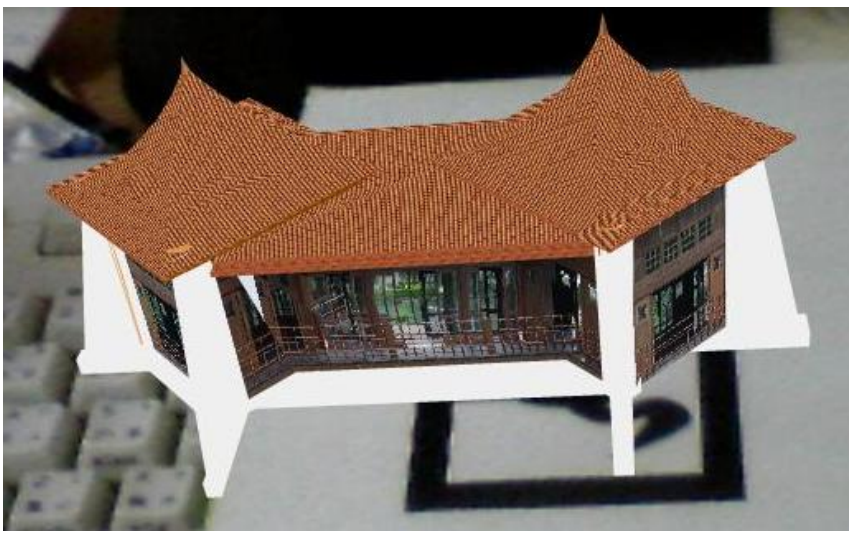

Fig. 6. The AR operation of the Mid-Lake Pavilion of Taichung Park

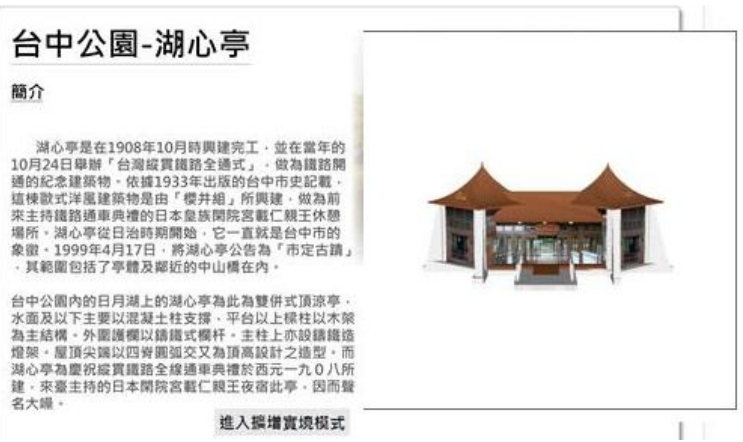

Fig. 7. The complete introduction web page of the Mid-Lake Pavilion of Taichung Park

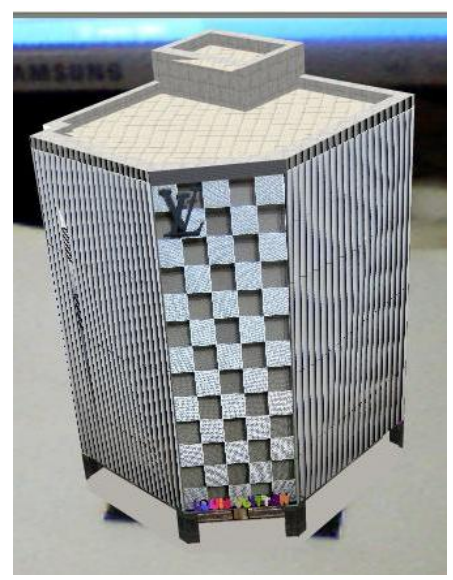

Fig. 8. The AR operation of one famous building in Taichung

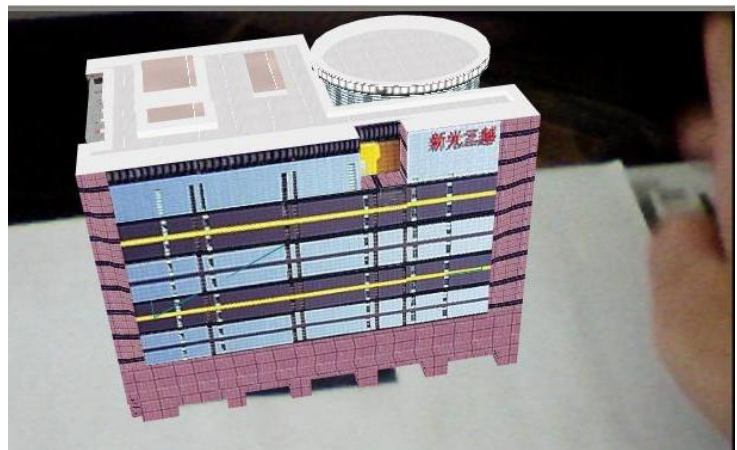

Fig. 9. The AR operation of the famous department building in Taichung

More famous buildings and parks are introduced with Augmented Reality effect on the web site, as shown in Figure 8 and Figure9. According to users' opinions, the interesting operation givens they lots of fun. As playing with the Augmented Reality effect, they read lots of introduction pages and know more interesting information about the city.

Another visual example is given as follows. The introduction of famous Taiwan food is also introduced in the website. The example is given in Figure 10 and Figure11. The rending design increases the visual effect of the web page. Most of the users who really like the visual design stay much longer on the webpage for reading the introduction of local famous food.

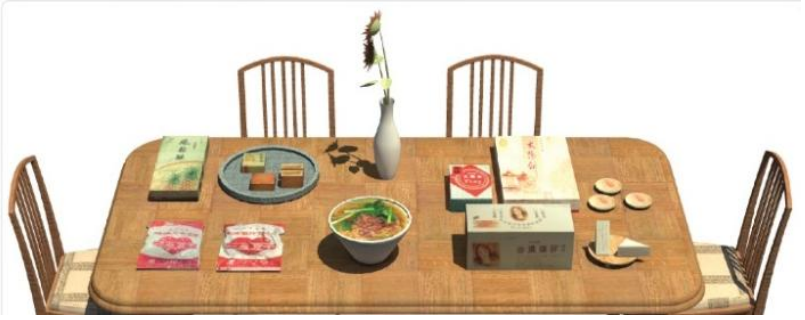

Fig. 10. The visual representtion of famous Taiwn food 




Fig. 11. The AR operation of Taiwan snack

\section{RESEARCH DISCUSSION}

To please users is not an easy job, but it is an important for keeping the web stickiness. However, a good web page designer has to rapidly cause the attention of users since web surfers have so much information available to them that they often get bored. In order to keep the user's attention, new technology is always a good try. Augmented Reality is such popular since the webcam and network become more convenient for PC users. The result is a rich combination of physical and virtual realities to increase the visual representation of web pages. The advantage is helpful for attracting the user's attention and interest. Therefore, the user stays longer on the page and possibly reads the supplied information on the website.

The first conclusion is that Augmented Reality technology increasing the visual web page design helps to attract the users' attention and increases the chance for users to read the information on the web pages. The visual representation in Augmented Reality is rapidly in progress currently and will become the main trend of mobile devices in the near future.

The second conclusion is that the 3D models have to be built closely to the real object. Do not cheat users with beautiful but fake models. Moreover, the third conclusion is that the easy operation of Augmented Reality technology is an important issue. Turn on the webcam automatically and shown the Augmented Reality effect quickly can help users continuously to enjoy the Augmented Reality application. Some users will give up since the system asks them to install the webcam or software.

During the research, we found the importance of attention management. Clear and concise graphics are the basic requirement of the web page design. Rendering design is such popular. However, some opinions imply that the 3D-like or visual model is sometimes embellished too much. It is easily cause the disapproval of users.

With Augmented Reality tasks the view of the real world behind the graphical annotations, and the interaction between the graphics and the real world, make Augmented Reality perception qualitatively different from anything previously studied. We will focus on the study of Augmented Reality technology for improving the representation skill. The user opinions are such important to increasing the opportunities of operations and business. Moreover, the application of Augmented Reality with smart phones is also interesting. To increase the user interactive operations for different experiences is always the research direction in the future.

\section{REFERENCES}

[1] R. Azuma, "A Survey of Augmented Reality," Presence: Teleoperators and Virtual Environments, August 1997, pp. 355-385.

[2] R. Azuma, Y. Baillot, R. Behringer, S. Feiner, S. Julier, and B. MacIntyre, "Recent advances in augmented reality," IEEE Computer Graphics and Applications, Vol.21, No.6, 2001, pp.34-47.

[3] D.A. Bowman and R.P. McMahan, "Virtual Reality: How Much Immersion is Enough?," IEEE Computer, Vol.40, No.7, 2007, pp.36-43.

[4] R. Bias, "The Pluralistic Usability Walkthrough: Coordinated Empathies," in J. Nielsen \& R. Mack Usability Inspection Methods, John Wiley, 1994, pp.63-76

[5] K. Börner, and C Chen,. (eds.) Visual Interfaces to Digital Libraries. Springer Verlag, Lecture Notes in Computer Science, Vol. 2539, 2002.

[6] D. Bowman, E. Kruijff, , J. LaViola, and I. Poupyrev, 3D User Interfaces: Theory and Practice, Addison Wesley, Boston, July 2004.

[7] D. Bowman, J. Chen, C. Wingrave, et al., "New Directions in 3D User Interfaces," International Journal of Virtual Reality, Vol.5, No.2, 2006, pp. 3-14.

[8] S. Card, J. Mackinlay, and B. Shneiderman(eds.), Readings in Information Visualization: Using Vision to Think. Morgan Kaufmann, 1999.

[9] A. Celentano, and F. Pittarello, "A Content Centered Methodology for Authoring 3D Interactive Worlds for Cultural Heritage," in D. Bearman, F. Garzotto (eds.), Proc. of Int. Cultural Heritage Informatics Meeting ICHIM'2001 (Milan, 3-7 September 2001), Cultural Heritage and Technologies in the Third Millennium, Vol. 2, 2001, pp. 315-324.

[10] T. Grossman, D. Wigdor and R. Balakrishnan, "Exploring and reducing the effects of orientation on text readability in volumetric displays," ACM CHI, 2007, pp. 483-492.

[11] J. L. Gabbard, J. E. Swan, D. Hix, S.-J. Kim, and G. Fitch, "Active text drawing styles for outdoor augmented reality: A user-basedstudy and design implications," Virtual Reality Conference, IEEE, 2007, pp.35-42.

[12] M. Hassenzahl, A. Beu and M. Burmester, " Engineering Joy,” IEEE Software, 2001.

[13] T. H“ollerer, S. Feiner, T. Terauchi, G. Rashid,and D. Hallaway, "Exploring MARS: Developing indoor and outdoor user interfaces to a mobile augmented reality system," Computers and Graphics, Vol.23, No.6, 1999, pp.779-785.

[14] C. Hsu, "The Feasibility of Augmented Reality on Virtual Tourism Website," The 4th International Conference on Ubi-media Computing, 2011.

[15] C. Lewis, P. Polson, C. Wharton, and J. Rieman, "Testing a walkthrough methodology for theory-based design of walk-up-and-use interfaces," In Proceedings of CHI 1990, pp.235-242.

[16] A. Kulik, "Building on Realism and Magic for Designing 3D Interaction Techniques," In IEEE Computer Graphics \& Applications, Vol.29, No.6, November/December 2009, pp. 22-33.

[17] F Liarokapis, "An augmented Reality Interface for Visualizing and Interacting with Virtual Content," Virtual Reality, Vol.11, No.1, 2007, pp. 23-43.

[18] W. Mackay and A. Fayard, "Designing Interactive Paper: Lessons from three Augmented," Reality Projects, 1999.

[19] P. Milgram, H. Takemura, A. Utsumi, and F. Kishino, "Augmented Reality: A Class of Displays on the Reality-Virtuality Continuum," Proceedings of Telemanipulator and Telepresence Technologies, SPIE Vol. 2351, 1994, pp.282-292.

[20] H. Neale, and S. Nichols, "Designing and Developing Virtual Environments: Methods and Applications," in Proc. of Visualization and Virtual Environments Community Club VVECC'2001 Workshop, Designing of Virtual Environments, 2001.

[21] J. Nielsen, and R. Molich, "Heuristic Evaluation of User Interfaces," In Proceedings of ACM CHI'90 Conference on Human Factors in Computing Systems, 1990, pp. 249-256. 
[22] J. Nielsen, "Heuristic Evaluation", In Nielsen, J. and Mack, R. L. (Eds.), Usability Inspection Methods, John Wiley and Sons, New York, 1994, pp. 25-62.

[23] J.-Y. Oh, W. Stuerzlinger and D. Dadgari, "Group Selection Techniques for Efficient 3D Modeling," In IEEE Symposium on 3D User Interfaces, 2006, pp. 95-102.

[24] M. Rachel, “Augmented Reality Is Finally Getting Real,” Technology Review, 2 August 2012.

[25] F. Steve, "Augmented reality: a long way off?" AR Week. Pocket-lint. Retrieved 3 March 2011.

[26] J. Vallino, Interactive Augmented Reality, PhD Thesis, Department of Computer Science, University of Rochester, 1998, New York, USA.

[27] C. Wingrave and J. LaViola, "Reflection on the Design and Implementation of Virtual Environments," In Special Issue of Presence: Teleoperators and Virtual Environments:Reflections on the Design and Implementation of Virtual Environment Systems, Vol.19, No.2, 2010.
[28] Google Sketchup, http://www.sketchup.com/product/newin7.html

\section{AUTHORS PROFILE}

Chouyin Hsu received the M.S. degree in Computer Science in 1993 from Ohio University, USA and the Ph.D degree in information management in 2006 from National Chiao Tung University, Hsinchu, Taiwan. She is currently an Associate Professor in the Department of Information Management at Overseas Chinese University, Taichung, Taiwan. Her current research interests include Data Mining, Visual User Interface Design, Project Management and Enterprise Resource Planning.

Haui-Chih Shiau received the M.S. degree in Information Management in 2010 from Overseas Chinese University, Taichung, Taiwan. He is currently a second-year graduate student in the Department of Information Technology at Overseas Chinese University Taichung, Taiwan. Her interests include Augmented Reality, 3D Unity, Mobile phone Application. 